\title{
THE SUM OF KIDNEY DONOR PROFILE INDEX AND ESTIMATED POST-TRANSPLANT SURVIVAL SCALE AND THEIR CORRELATION WITH EGFR DECLINE IN DECEASED DONOR KIDNEY RECIPIENTS
}

\author{
Pablo Maggiani-Aguilera ${ }^{1,2}$, Sergio Hernández-Estrada ${ }^{1,2 *}$, José H. Cano-Cervantes ${ }^{1,2}$, \\ Jonathan S. Chávez-IÑIguez ${ }^{3,4}$, Christian Pérez-Flores ${ }^{3,4}$, Odette del C. Díaz-Avedaño ${ }^{1,2}$, \\ María C. Oseguera-Vizcaíno ${ }^{3,4}$, Mayra Matías-Carmona ${ }^{1,2}$, Daniel F. Ovando-Morga ${ }^{1,2}$, \\ María G. Ramírez-Ramírez ${ }^{1,2}$, Guillermo Navarro-Blackaller ${ }^{3,4}$ Marco A. Covarrubias-Velasco 3,4 , \\ José A. Torres-Mayorga ${ }^{3,4}$, Benjamín Gómez-Navarro ${ }^{4}$, and Guillermo García-García ${ }^{3,4}$ \\ ${ }^{1}$ Department of Nephrology, Renal Transplant Unit, Centro Médico Nacional 20 de Noviembre, Institute for Social \\ Security and Services for State Workers (ISSSTE), Mexico City; ${ }^{2}$ Posgraduate Faculty, School of Medicine, Universidad \\ Nacional Autónoma de México (UNAM), Mexico City; ${ }^{3}$ Renal Transplant Unit, Civil Hospital of Guadalajara Fray \\ Antonio Alcalde, Guadalajara, Jal.; ${ }^{4}$ University of Guadalajara Health Sciences Center, Guadalajara, Jal., Mexico
}

\begin{abstract}
Background: The impact of donor quality on post-kidney transplant survival may vary by candidate condition. Objective: Analyzing the combined use of the Kidney Donor Profile Index (KDPI) and the estimated post-transplant survival (EPTS) scale and their correlation with the estimated glomerular filtration rate (eGFR) decline in deceased-donor kidney recipients (DDKR). Methods: This was a retrospective, observational cohort study. We included DDKRs between 2015 and 2017 at a national third-level hospital. Results: We analyzed 68 DDKR. The mean age at transplant was $41 \pm 14$ years, 47 (69\%) had sensitization events, 18 (26\%) had delayed graft function, and 16 (23\%) acute rejection. The graft survival at 12 and 36 months was $98.1 \%$ (95\% Cl 94-100) and 83.7\% (95\% Cl 65-100), respectively. The Pearson correlation coefficient between the percentage reduction in the annual eGFR and the sum of EPTS and KDPI scales was $r=0.61, p<0.001$. The correlation coefficient between the percentage reduction in the annual eGFR and the EPTS and KDPI scales separately was $r=0.55, p<0.001$, and $r=0.53$, $p<0.001$, respectively. Conclusions: The sum of EPTS and KDPI scales can provide a better donor-recipient relationship and has a moderately positive correlation with the decrease in eGFR in DDKR. (REV INVEST CLIN. 2021;73(4):216-21)
\end{abstract}

Key words: Kidney Donor Profile Index. Estimated post-transplant survival. Renal kidney graft survival. Deceased-donor kidney transplant. Estimate glomerular filtration rate. Graft loss.

*Corresponding author:

Sergio Hernández-Estrada

E-mail: sergiohernandezmed@hotmail.com
Received for publication: 18-12-2020

Approved for publication: 03-02-2021

DOI: $10.24875 / R I C .20000618$

0034-8376 / (c) 2021 Revista de Investigación Clínica. Published by Permanyer. This is an open access article under the CC BY-NC-ND license (http://creativecommons.org/licenses/by-nc-nd/4.0/). 


\section{INTRODUCTION}

Kidney transplantation provides the optimal kidney replacement therapy for the majority of people with endstage kidney disease. Although 1-year patient and graft survival now exceed $95 \%$ in major transplant centers, long-term outcomes have failed to improve overtime $^{1}$. One reason could be that kidneys that have been chosen for transplantation were not the optimal organs. For this reason, one of the current challenges is to achieve an adequate evaluation of the kidney's quality or viability, to reduce the discard rate of potential valid organs, and to allocate the deceased-donor kidney to the recipient where the organ will perform the best. The United Network for Organ Sharing added the Kidney Donor Profile Index (KDPI) to DonorNet ${ }^{\circledR}$ to evaluate offers and to make better and more informed decisions regarding which organs to accept. The Kidney Donor Risk Index (KDRI) combines a variety of donor factors to summarize the risk of graft failure after kidney transplant into a single number and expresses the relative risk of kidney graft failure for a given donor compared to the median kidney donor from last year. The KDPI maps the KDRI onto a percentage scale, ranging from 0 to $100 \%$. Lower KDPI values are associated with higher estimated quality donors and vice versa. KDPI is also used in the kidney allocation system (KAS).

Likewise, the estimated post-transplant survival (EPTS) score is assigned to all adult kidney candidates on the waiting list and is based on several factors (age, presence of diabetes mellitus, dialysis vintage, and previous history of solid organ transplant). A candidate's EPTS score can range from 0 to $100 \%$. A high EPTS score translates into shorter patient survival after transplant. The concept of longevity was established based on the fact that $20 \%$ of the best renal grafts (determined by the KDPI) will preferably be assigned to patients on the waiting list with the lowest EPTS scores $(0-20)^{2-4}$. Candidates with EPTS scores of $<20 \%$ will receive offers for kidneys from donors with similar KDPI scores before other candidates at the local, regional, and national levels of distribution ${ }^{5}$. Few studies have validated the performance of these scales inside and outside the United States ${ }^{6-8}$. One study analyzed the utility of these two scales in Mexico. Martínez-Mier et al. reported that the median survival was significantly higher in patients with EPTS < 20 compared to patients with EPTS > 20, and that for every 20\% EPTS increment, the patient's survival was lower' ${ }^{9}$. In our country, we do not have any validated and applied instrument to determine patient survival after a transplant. Our objective was to establish the use of the KDPI and EPTS scales and their correlation with the estimated glomerular filtration rate (eGFR) decline in deceaseddonor kidney recipients (DDKRs) and to assess the reproducibility of the organ assignment system of the USA in our population. To expand the use of marginal kidneys, we have originally added the sum of both scales and its correlation with a glomerular filtration rate decline, to characterize the interaction between donor quality and candidate condition, facilitating the use of such kidneys without sacrificing outcomes.

\section{METHODS}

\section{Data sources and study design}

In a retrospective, observational cohort study conducted between 2015 and 2017, we studied all DDKR at the national third-level Hospital 20 de Noviembre (Mexico City, Mexico), over 36 months following transplant; KDPI and EPTS scores were collected from clinical files, which were filled by the transplant staff. We excluded recipients of living kidney donors, multiorgan transplants, and patients aged $<18$ years at the time of transplant. In addition, we excluded patients with missing data for eGFR, KDPI, and EPTS scores, and those with primary graft dysfunction. All patients included were donors after brain death.

\section{Patient selection, group definitions, and study variables}

Demographic and clinical data, including age, gender, primary renal disease, sensitization events, peak panelreactive antibody, cold ischemia time, dialysis type and vintage, KDPI and EPTS calculator, delayed graft function, post-transplant diabetes mellitus, acute rejection, eGFR, and cause of graft loss, were collected from clinical notes and electronic records. The eGFR was calculated using the CKD-EPI equation ${ }^{10}$. The KDPI and EPTS were calculated on the website: (optn.transplant. hrsa.gov). The time on dialysis for patients with a second or third transplant was determined from the date of the last kidney graft loss (return to dialysis) to the time of retransplantation. Delayed graft function was defined as the need for dialysis in the immediate 7-day post-transplant period. Graft loss was confirmed by 
Figure 1. Cohort selection from National Medical Center 20 de Noviembre, Mexico.

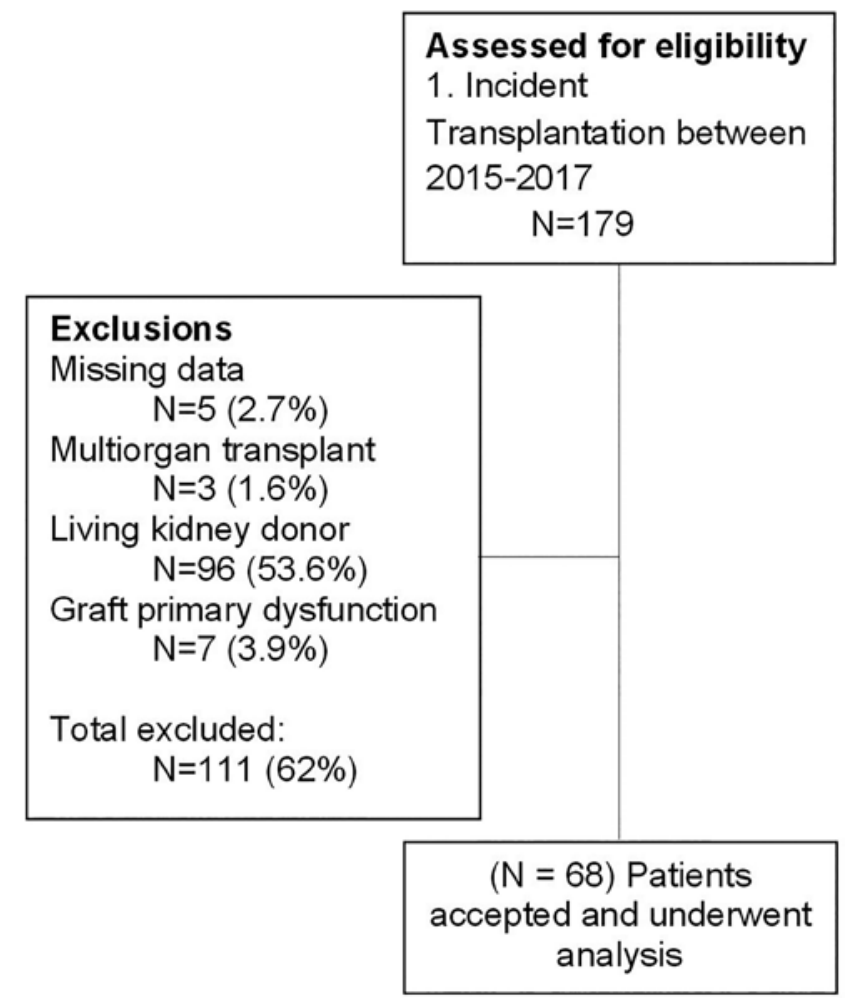

biopsy and non-response to medical or surgical treatment, with subsequent return to dialysis therapy. Acute rejection was diagnosed with a kidney biopsy and using Banff criteria. Furthermore, the death of the recipient with a functioning graft from causes related to his or her disease and transplantation was included. The study was approved by the Institutional Ethical Committee of the hospital. This protocol was written according to the Strengthening the Reporting of Observational Studies in Epidemiology guide ${ }^{11}$. The study was conducted in accordance with the Declaration of Helsinki.

\section{Study outcomes}

The primary outcome was to examine the sum of the $\mathrm{KDPI}$ and EPTS scales and their correlation with percentage reduction in the annual eGFR in DDKR. Secondary outcomes were the correlation between the percentage reduction in the annual eGFR and KDPI, EPTS, acute rejection, cold ischemia time, and delayed graft function, separately. The percentage reduction in the annual eGFR was calculated using the CKD-EPI equation between the $1^{\text {st }}$ month and the end of the $3^{\text {rd }}$ year or until graft loss.

\section{Statistical analysis}

Data are presented as mean \pm SD and count (\%). A Shapiro-Wilk test was used to determine the distribution of the variables. Overall graft survival at 36 months of follow-up after transplantation was assessed using the Kaplan-Meier method. Correlation between the different variables was calculated with the Pearson correlation coefficient. Analysis was performed using the R studio statistical software. Statistical significance was defined at $p<0.05$.

\section{RESULTS}

Between 2015 and 2017, a total of 179 patients underwent kidney transplantation; 5 (2.7\%) patients lacking data, $3(1.6 \%)$ patients with a multiorgan transplant, 96 (53.6\%) living kidney donor recipients, and $7(3.9 \%)$ with primary graft dysfunction, were excluded, for a total of 68 patients included in this report (Fig. 1). The mean age at transplant was $41 \pm$ 14 years; 47 (69\%) had sensitization events. Eighteen (26\%) patients had delayed graft function and 16 
(23\%) developed acute rejection. The mean cold ischemia time was $16 \pm 5 \mathrm{~h}$, and the mean KDPI and EPTS scores were $43 \pm 29 \%$ and $30 \pm 27 \%$, respectively (Table 1).

The $12^{\text {th }}$ and $36^{\text {th }}$ month graft survival was 98.1 (95\% Cl: $94-100)$ and $83.7 \%(95 \% \mathrm{Cl}: 65-100)$, respectively. The Pearson correlation coefficient between the percentage reduction in the annual eGFR and the sum of EPTS and KDPI scales was $r=0.61$, $p<0.001$ (Fig. 2A). The correlation coefficient between the percentage reduction in the annual eGFR and the KDPI and EPTS scales was $r=0.53, p<0.001$ and $r=0.55, p<0.001$, respectively (Fig. $2 B$ and $C$ ). The correlation coefficient between the percentage reduction in the annual eGFR and acute rejection, delayed graft function, and cold ischemia time was $r=0.21, p=0.08 ; r=0.19, p=0.12 ;$ and $r=-0.17$, $p=0.18$, respectively.

\section{DISCUSSION}

In this retrospective cohort of 68 DDKR, we found that the sum of the EPTS and KDPI scores had a moderately positive correlation with the decrease in eGFR, and that the sum exceeded the correlation observed between the scales separately. The utilization of KDPI and EPTS scores alone could have some limitations in selecting the appropriate kidneys and recipients. There is a report where, in candidates with low EPTS scores (e.g., < 40), the KDPI had a limited impact on survival benefit ${ }^{6}$. The authors concluded that the effect of KDPI on survival benefit was modified by EPTS, suggesting that the potential impact of marginal donor quality should be assessed according to the candidate's condition.

The best correlation observed between the sum of the two scales (KDPI + EPTS) concerning the loss of eGFR, opens the opportunity to create a tool that helps transplant specialists to improve the evaluation of kidney offers. Formulating a tool that considers the sum of both scales would improve the process of evaluating a kidney offer in several ways. First, it would take into account the specifications of each candidate, since the impact of kidney quality on the preservation of the eGFR varies substantially from the recipient's health status, and second, the simplicity of that model would open a possibility for shared decision-making.
Table 1. Baseline characteristics of deceased-donor kidney recipients from Centro Médico Nacional 20 de Noviembre, Mexico

\begin{tabular}{|c|c|}
\hline Variable $^{a}$ & Total \\
\hline Number of patients & 68 \\
\hline Age at transplant, years, mean (SD) & $41.5(14.3)$ \\
\hline \multicolumn{2}{|l|}{ Gender } \\
\hline Female & $28(41.1)$ \\
\hline Male & $40(58.8)$ \\
\hline \multicolumn{2}{|l|}{ Primary renal disease } \\
\hline Diabetic nephropathy & $9(13.2)$ \\
\hline GN & $5(7.3)$ \\
\hline Polycystic kidney disease & $6(8.8)$ \\
\hline Reflux nephropathy & $3(4.4)$ \\
\hline Hypertension & $3(4.4)$ \\
\hline FSGS & $3(4.4)$ \\
\hline Unknown & $39(57.3)$ \\
\hline Statin use prior transplant & $8(11.7)$ \\
\hline Sensitization events & $47(69.1)$ \\
\hline Blood transfusion & $33(48.5)$ \\
\hline Pregnancy & $9(13.2)$ \\
\hline Previous organ transplantation & $5(7.3)$ \\
\hline \multicolumn{2}{|c|}{ Peak panel-reactive antibody, mean (SD) } \\
\hline Class I & $3.2(6.4)$ \\
\hline Class II & $6.1(10.1)$ \\
\hline Cold ischemia time, hours, mean (SD) & $16.2(5.1)$ \\
\hline \multicolumn{2}{|l|}{ Dialysis type } \\
\hline Hemodialysis & $23(33.8)$ \\
\hline Peritoneal dialysis & $27(39.7)$ \\
\hline Both & $18(26.4)$ \\
\hline Dialysis vintage, months, mean (SD) & $83(45.8)$ \\
\hline KDPI score, mean (SD) & $43.6(29.7)$ \\
\hline EPTS score, mean (SD) & $30.7(27.8)$ \\
\hline \multicolumn{2}{|l|}{ Induction therapy } \\
\hline Thymoglobulin & $64(94.1)$ \\
\hline Basiliximab & $4(5.8)$ \\
\hline \multicolumn{2}{|l|}{ Maintenance therapy } \\
\hline Tacrolimus & $64(94.1)$ \\
\hline Cyclosporine & $4(5.8)$ \\
\hline Mycophenolic acid & $68(100)$ \\
\hline Sirolimus & $0(0)$ \\
\hline Azathioprine & $0(0)$ \\
\hline
\end{tabular}


Table 1. Baseline characteristics of deceased-donor kidney recipients from Centro Médico Nacional 20 de Noviembre, Mexico (continued)

\begin{tabular}{lc}
\hline Variable $^{a}$ & Total \\
\hline Maintenance therapy & \\
\hline Delayed graft function & $18(26.4)$ \\
Post-transplant diabetes mellitus & $2(2.9)$ \\
Acute rejection & $16(23.5)$ \\
Acute antibody-mediated rejection & $11(16.1)$ \\
Acute cellular rejection & $4(5.8)$ \\
Mixed rejection & $1(1.4)$ \\
eGFR at the $1^{\text {st }}$ month, mean (SD) & $77.4(23.8)$ \\
eGFR at the end of follow-up (36 & $61.9(28.8)$ \\
months), mean (SD) & $3(4.4)$ \\
Graft failure & $1(1.4)$ \\
\hline Cause of graft failure & $2(2.9)$ \\
\hline Need for RRT & \\
\hline Death censored graft loss
\end{tabular}

an (\%), unless otherwise specified.

KDPI: Kidney Donor Profile Index; EPTS: estimated post-transplant survival; eGFR: estimated glomerular filtration rate; RRT: renal replacement therapy; GN: glomerulonephritis.
Several recent studies have proposed statistical tools to support clinicians in making decisions on kidney offers. Bae et al. ${ }^{6}$ created an online tool (www.transplantmodels.com/kdpi-epts) that offers interactive visualization of the impact of KDPI on survival benefit for a specific value of EPTS. Wey et al. ${ }^{12}$ created a tool to predict if a candidate who declined a kidney offer would subsequently receive a kidney transplant and maintain a functioning graft at 3 years after declining the initial kidney offer.

Compared to these approaches, creating a tool that includes the sum of both scales would be simple to use and could be a good option to predict eGFR decline. Therefore, observing the donor and the recipient as a whole and not separately expresses best the reality of transplants and could be an interesting new tool that helps us predict more precisely the deterioration of the eGFR in DDKR. In addition, we were also able to observe that acute rejection, cold ischemia time, and delayed graft function did not show a better correlation than the sum of the KDPI and EPTS scales regarding eGFR decrease.

Figure 2. Correlation coefficient between the percentage reduction in the annual estimated glomerular filtration rate and different scores. (A) The sum of estimated post-transplant survival (EPTS) and Kidney Donor Profile Index (KDPI) scales. (B) KDPI scale. (C) EPTS scale. Confidence interval in light gray shadow.
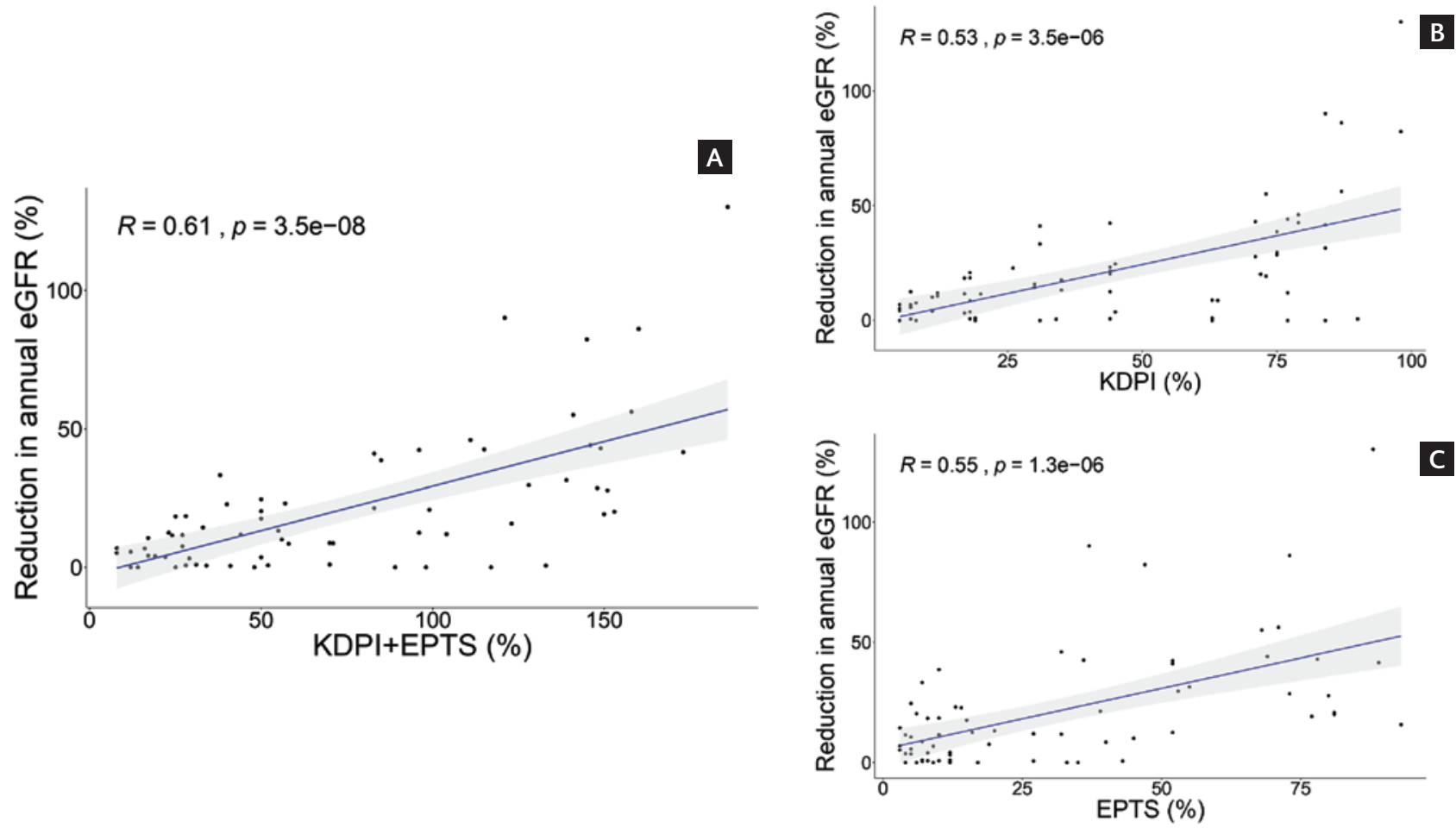
Our study has some limitations. The observational and retrospective design and the relatively small sample size require validation of our results with a larger sample size and the inclusion of more transplant facilities. A strength worthy of mentioning is that our study is the first to combine the sum of two common scores to improve the prediction of the course of eGFR overtime.

In conclusion, sum of EPTS and KDPI scales can provide a better donor-recipient relationship and has a moderately positive correlation with the decrease in eGFR in DDKR, supporting a new tool for trials in kidney transplantation.

\section{ACKNOWLEDGMENTS}

The authors thank the medical, nursing, and associate health staff of the Nephrology Service at the Centro Médico Nacional 20 de Noviembre, from the ISSSTE, for treating all patients with joy and kindness.

\section{REFERENCES}

1. Clayton PA, Lim WH, Wong G, Chadban SJ. Relationship between eGFR decline and hard outcomes after kidney transplants. J Am Soc Nephrol. 2016;27:3440-6.
2. Ramanathan R, Gupta G, Kim J, Quinn K, Behnke M, Kang L, et al. Retroactive application of the new kidney allocation system to renal transplants performed in the ECD/SCD era. Clin Transplant. 2015;29:1148-55.

3. Stewart DE, Klassen DK. Early experience with the new kidney allocation system: a perspective from UNOS. Clin J Am Soc Nephrol. 2017;12:2063-5.

4. Wang CJ, Wetmore JB, Israni AK. Old versus new: progress in reaching the goals of the new kidney allocation system. Hum Immunol. 2017;78:9-15.

5. Israni AK, Salkowski N, Gustafson S, Snyder JJ, Friedewald J], Formica RN, et al. New national allocation policy for deceased donor kidneys in the United States and possible effect on patient outcomes. J Am Soc Nephrol. 2014;25:1842-8.

6. Bae S, Massie AB, Thomas AG, Bahn G, Luo X, Jackson KR, et al. Who can tolerate a marginal kidney? Predicting survival after deceased donor kidney transplant by donor-recipient combination. Am J Transplant. 2019:19:425-33.

7. Chopra B, Sureshkumar KK. Changing organ allocation policy for kidney transplantation in the United States. World J Transplant. 2015:5:38-43.

8. Schold JD, Buccini LD, Reese PP, Poggio ED, Goldfarb DA. Effect of dialysis initiation for preemptively listed candidates in the revised kidney allocation policy. Am J Transplant. 2014;14: 2855-60.

9. Martínez-Mier G, Soto-Miranda E, Moreno-Ley PI, Méndez-López MT, Budar-Fernández LF, Ávila-Pardo SF, et al. Escala de sobrevida de pacientes después de trasplante renal de donantes fallecidos en Veracruz, México. Rev Med Inst Mex Seguro Soc. 2019:57:149-55.

10. Levey AS, Stevens LA, Schmid CH, Zhang YL, Castro AF, Feldman, et al. A new equation to estimate glomerular filtration rate. Ann Intern Med. 2009;150:604-12.

11. Von Elm E, Altman DG, Egger M, Pocock SJ, Gotzsche PC, Vandenbroucke JP. The strengthening the reporting of observational studies in epidemiology (STROBE) statement: guidelines for reporting observational studies. Lancet. 2007;370:1453-7

12. Wey A, Salkowski N, Kremers WK, Schaffhausen CR, Kasiske BL, Israni AK, et al. A kidney offer acceptance decision tool to inform the decision to accept an offer or wait for a better kidney. Am J Transplant. 2018;18:897-906. 